https://doi.org/10.5719/aub-g/68.1/5

\title{
L'AMÉLIORATION URBAINE, UNE ACTION PLURIELLE POUR RECHERCHER LA QUALITÉ DE VIE. QUELS EFFETS SUR LA POPULATION ET LE CADRE DE VIE DANS LES ENSEMBLES COLLECTIFS D'ANNABA (NORD-EST ALGÉRIEN)
}

\author{
HAYET MEBIROUK ${ }^{1}$
}

\begin{abstract}
Résumé
L'amélioration du cadre et des conditions de vie en milieu urbain constitue l'enjeu de toute action politique. En Algérie cette question s'entreprend soit par le développement et l'amélioration de la qualité des services publics (transports publics) et des infrastructures (grands projets pour les métropoles), soit par les opérations d'amélioration urbaine qui s'inscriventdans le cadre d'un programme national ambitieux de soutien à la relance économique. Initié par l'État, ceprogramme vise la requalfication des périphéries urbaines et la réhabilitation des zones d'habitat dégradé et ce par l'aménagement deleurs espaces extérieurs et l'amélioration du cadre de vie.

Les programmes d'amélioration urbaine ont, plus ou moins, contribué à la réhabilitation de l'image de plusieurs cités d'habitat collectif d'Annaba. C'est dans une perspective de rendre compte de ces opérations et leurs effets sur le cadre de vie, de définir leurs atouts ainsi que leurs faiblesses sur le plan socio-spatialet environnemental que s'inscrit notre travail. Dans cette visée, nous avons mené un travail de terrain privilégiant l'observation directe complétée par le recueil de données auprès des services concernés et l'enquête par entretien directif. Des matériaux à partir desquels les constats de synthèse qui suivent sont tirés.
\end{abstract}

Mots-clés: Amélioration urbaine, qualité de vie, espace public, ensemble collectif, Annaba.

\section{Introduction}

La notion de qualité de vie en milieu urbain « fut évoquée pour la première fois dans le traité sur l'architecture et le bien-être de Léon Bastita Alberti, De re aedificatoria connu dès 1452 »(El-Kadi, 1997 : 35). Aujourd'hui, cette notion multidimensionnelle se trouve au cœur des projets d'aménagement et de développement, et s'impose en tant que revendication collective du bienêtre portée par la société et en tant que promesse politique engageant à la fois l'État et les collectivités territoriales (Barbarino, 2005). Demeurant un enjeu de

1 Maitre de Conférences à l'Institut de Gestion des Techniques Urbaines, Université Constantine 03. (+) 213 772801814. Email: hayat.mebirouk@univ-constantine3.dz 
taille, la qualité de vie fait référence à plusieurs notions dont celle de l'amélioration des conditions et du cadre de vie reconnuecomme déterminante de l'équilibre physique et psychologique des citadins (Lamri, 2012).

En Algérie, cette question remonte aux années 1995 avec la promulgation de l'instruction ministérielle $\mathrm{n}^{\circ} 89$ du 24 juin 1995 relative à la décentralisation de la gestion des opérations d'études et d'aménagement urbain. L'objectif de cette opération étatique est de réhabiliter les zones d'habitat précaire en prenant essentiellement la forme de régularisation de la propriété foncière et l'investissement par l'État dans les infrastructures de viabilité. Il s'agit, en fait, d'une amélioration des conditions de viabilité des quartiers sous-équipés notamment ceux résultant d'un développement urbain spontané dont l'intégration aux tissus urbains planifié est devenu nécessaire (Mebirouk, 2018). On pourrait croire ici que la recherche d'un cadre de vie agréable au sein des ensembles d'habitat collectif a émergé en 2001avec le redressement des prix de pétrole. A partir de cette date, l'Algérie a connu le début d'une embellie financière qui a suscité le lancement des opérations publiques d'amélioration urbaine s'articulant autourde plusieurs thèmes : la reconquête spatiale, l'aménagement urbain, la valorisation des espaces publics, la redynamisation économique du quartieret notamment l'intégration sociale des habitants à la ville.

La prédisposition des autorités publiques pour la réussite de ce programme s'est traduite par l'adoption de nouvelles lois dont les principales sont la loi n 06-06 du 20 février 2006 portant loi d'orientation de la ville promulguée dans un souci majeur celui d'une politique cohérentepermettant l'améliration du cadre de vie du citoyen (art 10 de la loi), la promotion des espaces publics et des moyens de transports en vue de faciliter la mobilité urbaine.

Le suvi de la mise en œuvre de cette politique est assigné à l'Observatoire national de la ville créé notamment pour élaborer des études sur le développement des villes dans le cadre de la politique nationale d'aménagement du territoire (art. 26 de la loi d'orientation de la ville). S'y ajoute, la loi ${ }^{\circ}$ 07-06 du 13 Mai 2007 ayant pour objet de définir les règles de gestion, de protection et de développement des espaces verts dans le cadre du développement durable (art 1 de la loi $\mathrm{n}^{\circ} 07-06$ ).

L'amélioration urbaine est ainsi, un programme national voire gouvernemental ambitieux s'inscrivant dans le plan de soutien à la relance économique. Ce plan d'action multisectoriel a tenté d'assurer la requalification des zones périphériques par la mise en œuvre d'opérations de réhabilitation et de reconquête spatiale. Du fait, l'objectif de ce travail est d'examiner ce programme qui semble avoir contribué à l'évolution du cadre de vie de plusieurs quartiers et l'amélioration de leur image. Cet objectif se réalise par l'analyse du produit fini dans certains quartiers de la ville d'Annaba en se

2 Il est à noter que cette loi n'a pas jusqu'à ce jour de décret d'application. 
référant d'une part au guide de mise en œuvre des travaux d'amélioration urbaine, de requalification du cadre bâti et de réhabilitation des espaces libres élaboré (en 2007) par le Centre National des Etudes et Recherches Intégrées du Bâtiment (CNERIB); et d'autre part à l'enquête de terrain en interrogeant quelques-uns des habitants sur un projet qui se veut socialpar l'intégration et l'implication de la population résidente à sa concrétisation.

\section{Méthodologie d'approche}

Pour répondre à cette problématique, nous nous sommes intéressés aux ensembles de logements collectifs d'Annaba qui constituent un excellent terrain d'investigation de la question car ils font partie des principaux quartiers sur lesquels ont été lancées les premières opérations d'amélioration urbaine. Cette typologie est le fruit de la procédure des ZHUN (Zones d'Habitat Urbain Nouvelles) engagée dans les années 1970, une procédure équivalente à celle des Zones à Urbanisation Prioritaire (ZUP) mises en place en France au cours des années 1960. Ce dispositif constitue une réponse adaptée aux besoins en logement d'une population de plus en plus nombreuse.

$\mathrm{Du}$ fait que nous partions d'une situation concrète comportant une problématique que nous ambitionnons d'évaluer, la méthode d'analyse retenue recourt à une technique de recherche qualitative dont les instruments sont :

- l'observation visuelle du projet réalisé (espaces et aménagements), cet instrument est important pour évaluer les comportements des citadins vis-à-vis de l'espace produit et la qualité de mise en œuvre des opérations d'aménagement;

- le recueil de données auprès des services concernés : direction de l'Urbanisme, de l'architecture et de la Construction (DUAC); direction de l'Hydraulique, etc.

- et l'enquête par entretien de type semi-directif pour que la personne interrogée puisse répondre avec plus de liberté. Bien que l'échantillon de personnes soit réduit (environ 100 personnes) nous le considérons comme significatif car composé de personnes d'âge, de sexes et de niveau socio-économique différents (femmes, enfants, commerçants, retraités, fonctionnaires, enseignants universitaires et visiteurs). Les questions retenues sont centrées sur la qualité de l'espace public, une question suffisamment développée au cours des années 1980 par des travaux de recherche notamment ceux qui ont abordé la problématique $\mathrm{du}$ renouvellement urbain dans les grands ensembles.

- Inspirées de la grille de lecture de Sablet de (1992), les principales questions portent sur l'attractivité urbaine, le besoin des habitants en matière d'espaces, le paysage végétal, le mobilier urbain et les 
matériaux de mise en œuvre, le sentiment de sécurité, l'implication de l'usager dans le processus d'aménagement des espaces extérieurs, la satisfaction apparente des usagers. Il est vrai que la grille de lecture remonte aux années 1990 mais la situation actuelle des espaces publics dans les ensembles collectifs algériens et le besoin en espace sont, pour ainsi dire comparables à ceux des pays développés des années 1960-1970.

\section{Les espaces publics des ensembles collectifs d'Annaba, de l'abandon au regain d'intérêt}

Les zones périphériques d'Annaba disposent-elles d'espaces publics ? La question peut paraitre banale si on se base sur une définition physique de l'espace ou si on se livre au jeu des opposés : tout ce qui est libre et tout ce qui n'est pas privé constituent l'espace public. Pourtant, cette question reste extrêmement féconde si on se réfère à l'aménagement, à l'usage et à l'appropriation de ces espaces (Navez-Bouchanine, 1992). Ce type de question étant posépour affirmer que jusqu'aux années 2000, les espaces publics caractérisant les ensembles collectifs d'Annaba étaient dépourvus du moindre aménagement et ne répondaient pas aux aspirations des usagers (Mebirouket al., 2005). Ces espaces se contentaient le plus souvent des miettes d'un espace figé par la voirie et les volumes bâtis, leur définition résiduelle a suscité chez le citoyen un sentiment d'insécurité et de morosité. Laissés à l'abandon plus de deux décennies (1974-2000), ces espaces deviennent à partir de l'année 2001 l'enjeu des pouvoirs publics en étant reconnus comme un élément fondamental dans le processus de revitalisation urbaine.

L'espace public n'est plus perçu comme « vide » entre deux entités construites, son aménagement devient un véritable enjeu social méritant toute l'attention. En réponse à cet enjeu, un programme de mise à niveau du tissu urbain a touché environ 3693 quartiers à travers le territoire national dont 762 constructions de type tissu spontané ; 1092 lotissements ; 938 grands ensembles. Ce programme présidentiel et gouvernemental a été mené sur l'ensemble du parc national du logement pour une durée s'étalant sur quatre ans.

La wilaya d'Annaba située au Nord-Est Algérien a bénéficié en 2007 de deux opérations d'amélioration urbaine pour une autorisation de programme de 235 millions de dinars (environ 1370000 Dollars) (El-Annabi, 2007). Les sites touchés par cette opérationsont situés au Nord-Est (El-Fakharine et El-Moukawama), à l'Ouest (Plaine-Ouest : Cité1172 logements, Cité687 logements et cité 1028 logements ; et cité 08 Mai 1945), et au Sud-Est (Seybous) (Figure 1). 


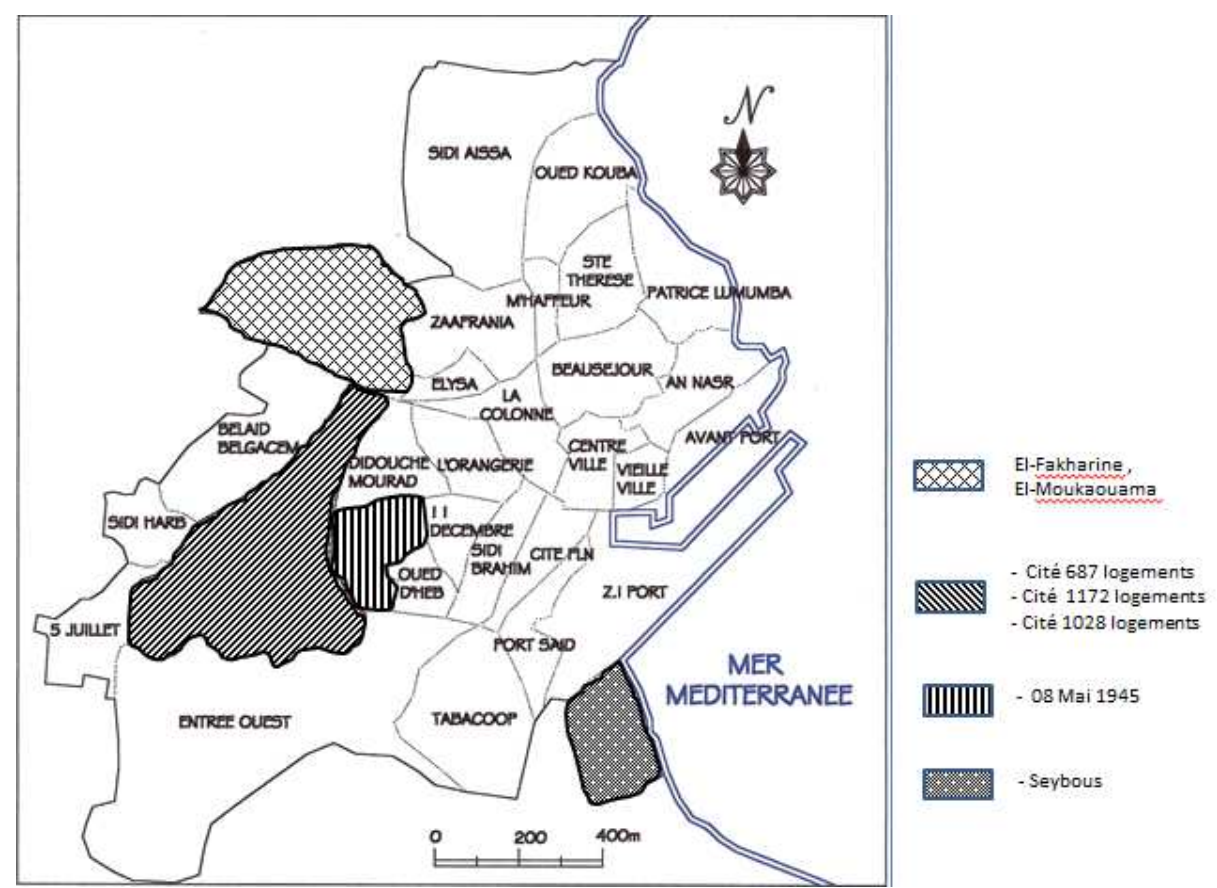

Fig. 1. Les sites touchés par l'amélioration Urbaine au titre de l'année 2007 Source : Auteur, 2018

Dans les quatre sites retenus, les interventionssont centrées sur les espaces extérieurs par leur aménagement (création des aires de jeux, aires de stationnement et espaces verts), sur le mobilier urbain par la réalisation des bancs et éclairages publics, et sur la desserte et les réseaux divers par leur renforcement. Considérant que la dernière intervention est une solution technique qui résout dans un premier temps les problèmes d'alimentation en eau potable, de raccordement en énergie électrique, d'assainissement des eaux usées et ménagères, et des eaux pluviales, et dans un deuxième temps les trottoirs et allées piétonnes et la réhabilitation de la voirie urbaine. Après trois ans (2010), Annaba a profité de soixante-deux opérations concernant plusieurs tissus urbains de l'époque coloniale (14 opérations) et postcoloniale. En cette date, l'attention des pouvoirs publics s'est tournée vers l'habitat collectif en lui consacrant 27 opérations (environ $44 \%$ du total) contre 35 destinées au reste des typologies. Dans cette optique, les cités d'habitat précaire ${ }^{3}$ suivis de l'habitat

3 Les quartiers d'habitat précaire ont connu (entre 1995-2000) un projet de résorption d'habitat précaire (RHP) initié par l'Etat sous l'impulsion du financement de la Banque Mondiale et destiné à améliorer la qualité de vie des habitants. Toutefois ce projet s'est soldé par un échec au vu d'un financement insuffisant et d'un encadrement peu qualifié. 
individuel sont ceux qui bénéficient le moins de ce projet Etatique avec seulement 03 opérationspour les quartiers en difficulté et 05 pour le lotissement (Figure 2).

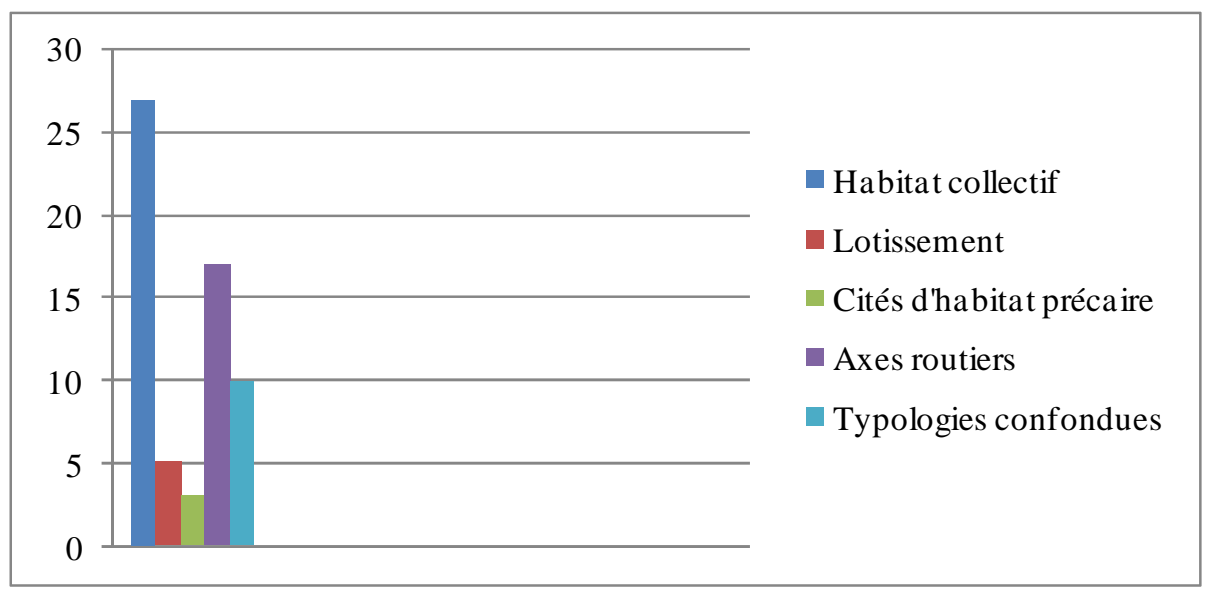

Fig. 2. Opérations d'amélioration urbaine au titre de l'année 2010 (DUC, 2010)

Il est possible de constater que les premiers sites d'habitat collectif choisis pour concrétiser le programme d'amélioration urbaine sont situés à l'Ouest de la ville. Il y va de la cité «Plaine-Ouest ${ }^{4}$ etcelle de «Oued-Edheb ${ }^{5}$ lesquelles ont bénéficié de dix opérations d'aménagement en plus de celles achevées avant 2007 (Figure 3). Réalisées au début des années 1980 dans le cadre des extensions urbaines de type habitat collectif, ces citéstirent leur nom soit de l'opération réalisée (cités 687 logements, 1172 logements, 1028 logements, etc.) soit de l'entreprise de réalisation (SONATIBA ${ }^{6}, \mathrm{OPGI}^{7}$, Géni-Sider, etc.). Le plus remarquableest que ces citésfurent reconnuespar des espaces extérieurssouvent imprécis tant dans leur délimitation physique que dans leur usage où se superposaient et coexistaient des pratiques urbaines et rurales (vente des articles à même le sol, pratiques ménagères, jardinages, jeux, etc.). L'espace vert, tel que précisé par Mebirouk (2008 : 35), occupe des délaissés de terrains et « constitue un dérivatif à la mauvaise conception d'un habitat omettant luimême les multiples besoins et aspirations de ses usagers ». A l'exception de la verdure spontanée voulant s'imposer comme dispositif d'embellissement des espaces non-bâtis aucun espace vert ne semble être manifestement prévu. Tant et si bien que les équipements d'agréments qui devaient l'accompagner

4 C'est une plaine située à l'ouest de la ville connue par le nom de Saf-Saf 1 et 2 (peuplier) séparé par un oued.

Le site est traversé par un Oued dit Edheb: Rivière d'Or.

6 Société Nationale de Bâtiments

7 Office de Promotion et de Gestion Immobilière 
systématiquement (bancs de repos, aires de jeux pour enfants, éclairages, etc.) n'existaient pas ou sont quasiment détériorés.

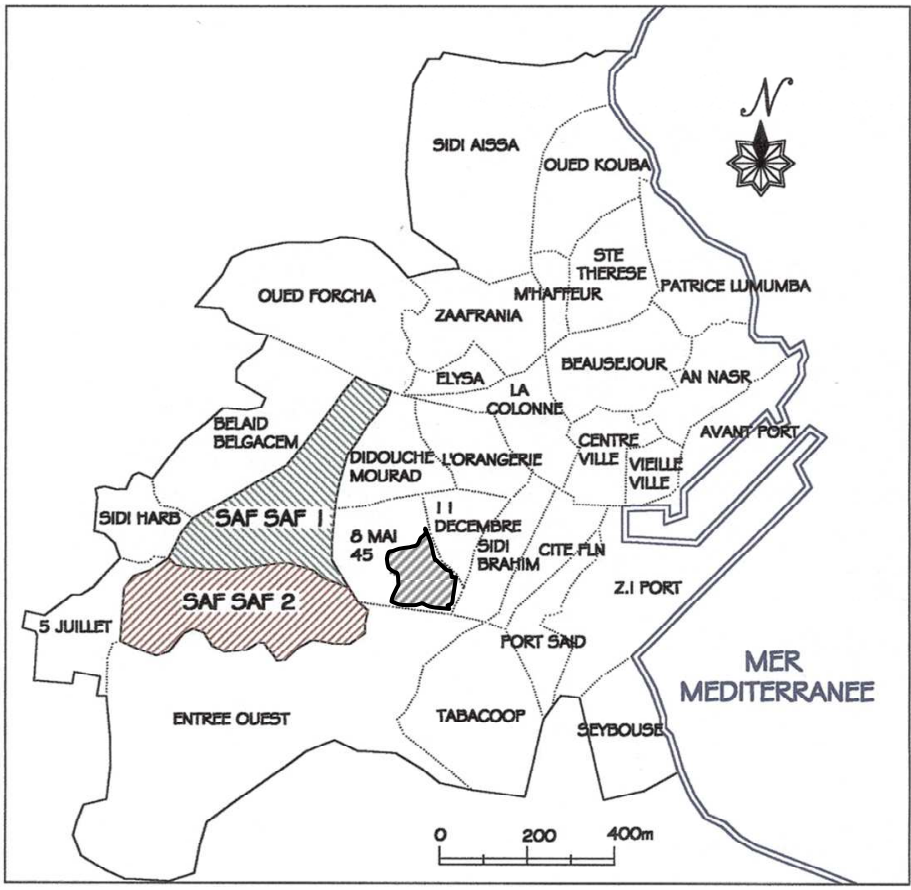

Oued-Dheb

Fig. 3. Localisation des cités d'habitat collectif : Plaine-ouest et Oued- Edheb Source : Auteur

Il est important de mentionner que la problématique des espaces publics a été largement condamnée par les travaux de recherche (Mebirouk, 2002, Zahi, 2012, Bahri, 2014) lesquels ont tenté de mettre en scène la négligence de ces espaces et de leur entretien par les autorités publiques. Il appert que la situation financière favorable de l'Algérie (2001) a sans nul doute fait naitre une volonté de repenser les espaces extérieurs dans les ensembles de logements collectifs par la réhabilitation et l'amélioration du cadre et des conditions de vie des habitants. Or la question qui se pose est de savoir si le produit fini dans le cadre de ce programme d'absorption des déficits d'aménagement urbain est acceptable d'autant qu'à travers ce programme l'État a tenté de rechercher une réponse durable en combinant trois aspects social, économique et environnemental. Dans cet ordre d'idée, l'aspect social se définit par la création des associations activant notamment dans le cadre de vie, l'environnement et les conditions de vie à la fois matériels et immatériels de la population. L'aspect économique se rapporte à l'animation de l'activité économique et la création d'emplois à 
travers les opérations lancées alors que l'aspect environnemental est consubstantiel de la rénovation des réseaux d'assainissement existants et aussi de l'aménagement et la création des espaces verts.

\section{Quels acteurs pour la mise en ouvre des opérations urbaines?}

Hormis l'instruction ministérielle $n^{\circ} 89$ du 24 juin 1995 susmentionnée, l'amélioration urbaine ne fait référence à aucune loi. C'est une opération régie par des correspondances ministérielles et des directives du wali appliquées par les directions déconcentrées. Cette opération est exécutée sur la base d'un document établi par le Centre National des Etudes et Recherches Intégrées du Bâtiment (CNERIB) sous la Tutelle du Ministère de l'Habitat et de l'Urbanisme. Le document prescrit toutes les dispositions visant à assurer la sécurité, la stabilité, l'hygiène et le niveau de confort conformément aux exigences normatives, environnementales et sociales.

Le programme d'amélioration urbaine est une action Étatique décidée à l'échelle centrale et mise en œuvre à l'échelle locale par la wilaya et ses organes déconcentrés. Théoriquement, le programme est conduit par trois sphères (Figure 4).

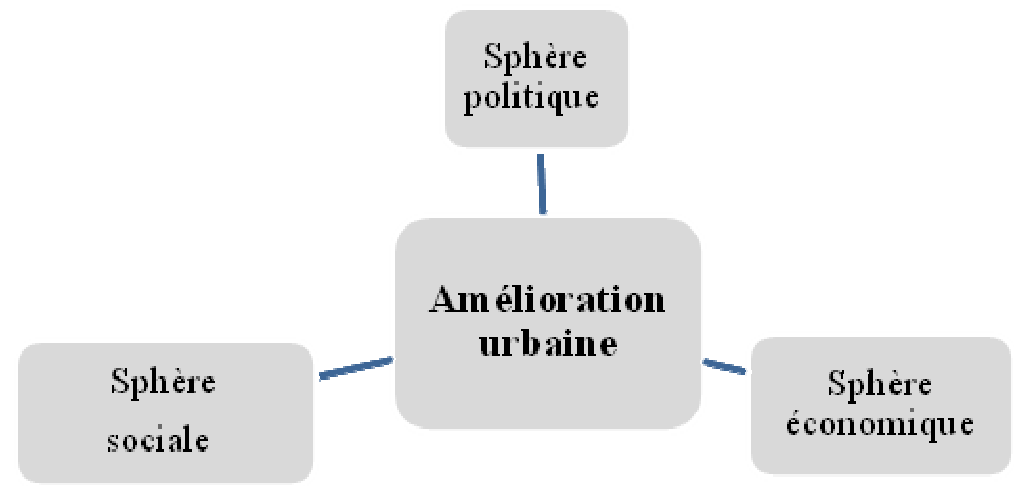

Fig. 4. Les sphères de l'opération d'amélioration urbaine Source : Auteur, 2018

\section{Les acteurs de la sphère politique}

\section{Les collectivités locales : La wilaya}

La Wilaya (Préfecture) est une collectivité publique territoriale dotée de deux organes : l'Assemblée Populaire de Wilaya «APW », et le Wali (Préfet). Représentant de l'Etat et délégué du Gouvernement au niveau de la wilaya, le 
Wali est chargé d'exécuter les décisions et les instructions reçues de chacun des ministres. Il anime, coordonne et contrôle l'activité des services de l'Etat chargés des différents secteurs d'activités dans la wilaya (Loi n $90-09$ de 1990).

\section{La Direction de l'Urbanisme, de l'Architecture et de la Construction (DUAC)}

C'est un service extérieur du ministère de l'habitat et de l'urbanisme chargé de mettre en ouvre au niveau locale la politique de l'urbanisme, de l'architecture et de la construction (Art. 3 du décret $n^{\circ} 13-13$ de 2013). Cette direction assure multiples missions dont les principales consistent à organiser les interventions de revitalisation au sein des tissus urbaine existants, à promouvoir un cadre bâti harmonieux et esthétique en fonction des caractéristiques géographiques, climatiques et sociale locales, et à assurer la cohérence dans le développement de l'habitat, des équipements publics, des services et des activités. Dans le domaine de construction, cette direction est chargée d'appliquer les documents techniques et réglementaires ainsi que les normes de construction en vigueur.

\section{Les acteurs de la sphère économique : Le professionnel et l'entreprise de réalisation}

Ce sont les agences ou bureaux d'études ainsi que les entreprises de réalisation désignés par la DUAC et la Wilaya pour la conception puis la réalisation du projet. Les critères de choix des bureaux d'études et des entreprises s'élaborent en fonction du critère du moins disant. En effet les projets d'architecture et d'urbanisme sont toujours considérés comme produits financiers et que le moins disanten étude ou en réalisation, selon le travail de recherche élaboré par SidiSalah-Nasri (2010), disposera des meilleures chances d'être retenu. Dans certains cas le budget de l'opération est fixé a priori avec un montant ne pouvant pas couvrir les frais du bureau d'études ou de l'entreprise désignés à cet effet.

Il est à noter que l'Office de Promotion et de Gestion Immobilière « OPGI » en tant qu'opérateur public, est chargé de la maitrise d'ouvrage des opérations d'assainissement (vides sanitaires) et de restructuration d'immeubles (ravalement des façades, reprise de l'étanchéité et lot peinture, etc.) dans le cadre du programme de réhabilitation.

\section{Les acteurs de la sphère sociale : Les habitants}

Comme nous l'avons mentionné plus haut, l'objectifdu programme d'amélioration urbaine est la reconquête des territoires périphériques. Ce projet 
se concrétise en prenant en compte la dimension sociale en tant qu'objectif principal. Il est, à première vue, fondé sur le partenariat entre l'État et la population locale, et vise à conforter plusieurs options notamment celle de l'implication responsable des populations locales par leur participation (Zahi, 2012). Pour ce qui est des textes législatifs, la loi d'orientation de la ville stipule dans son article 17 que les citoyens sont associés aux programmes relatifs à la gestion de leur cadre de vie. A cet effet, les pouvoirs publics sont appelés à conjuguer les conditions ainsi que les mécanismes qui permettent d'associer effectivement le citoyen aux programmes et actions liés à leur quartier.

Suivant ce principe, il est possible de croire que l'opération d'amélioration urbainedoit émaner d'un effort collectif par l'implication du citoyenà une action plurielle afin de retrouver la cohérence de la villeen général et de ses espaces publics en particulier. Une telle implication est traduite, d'après Mebirouk (2018) par la prise en charge de son avis dans les différentes phases de réalisation des aménagements et surtout dans l'entretien de ces aménagements et des espaces collectifs rattachés à son habiter.

A la différence de ce qui a été avancé et en se référant à l'enquête de terrain, les acteurs de l'opération d'amélioration se réduisent aux sphères politique et économique. Selon les affirmations des personnes interrogées (sexes et tous statuts confondus), l'habitant étant considéré comme un acteur « invisible » puisqu'il était exclu de la proposition d'aménagement etdu processus de mise en œuvre de ces opérations. Mais en dépit de son exclusion, l'habitant a accueilli ce projet avec satisfaction, les personnes interrogées attestent toutes que le programme a amélioré considérablement l'image de leur quartier par le ravalement des façades, l'aménagement des espaces extérieurs, la création des aires de jeux et la réalisation du mobilier urbain.

\section{Les effets du projet sur l'habitant et son cadre de vie}

Pour tirer les enseignements tant positifs que négatifs de ce projet d'amélioration urbaine, il nous semble fondamental de faire appel au processus de retour d'expérience comme démarche d'analyse a postériori de la gestion du projet. A dire vrai, ce projet a dégagé une série d'actions urgentes privilégiant un compromis entre besoins, objectifs et moyens disponibles selon un calendrier allant du court au long termes. Etant achevées dans la plupart des cités d'habitat collectif, les actions inscrites dans le court terme : sécurité, confort sanitaire, hygiène urbaine, aménagement des espaces communs, création des espaces de détente, etc. semblent parvenir à redorer l'image des quartiers réhabilités. Nous tentons dés lors d'évaluer le projet fini dans certains quartiers conformément aux objectifs fixés par l'opération urbaine : un premier objectif lié à la sécurité et l'hygiène à travers les travaux d'exécution de la voirie, les réseaux divers et 
l'aménagement des espaces vertset un second objectif lié au confort sociospatial et à la création d'un environnement agréable.

\section{La sécurité et l'hygiène}

\subsection{La réalisation de voies de desserte et de leur accessoire}

Il est bien connu que les travaux de voirie portent essentiellement sur l'ensemble des ouvrages réservés à la circulation de tous les véhicules, des deux roues et des piétons, ainsi que sur les aires de stationnement. Ces travaux, généralement complétés par la réalisation de parcs de stationnement, doivent contribuer à améliorer l'aspect du paysage, qu'il soit urbain ou rural.

Faisant abstraction des exigences techniques élaborées par le CNERIB, des imperfections ont été constatées dans la réalisation de certaines voiries et de leurs accessoires dans les cités Plaine-Ouest et Oued-D'heb (voie piétonne, trottoir, aires de stationnement) liées le plus souvent aux moyens de mise en œuvre (Figure 5, 6 et 7).
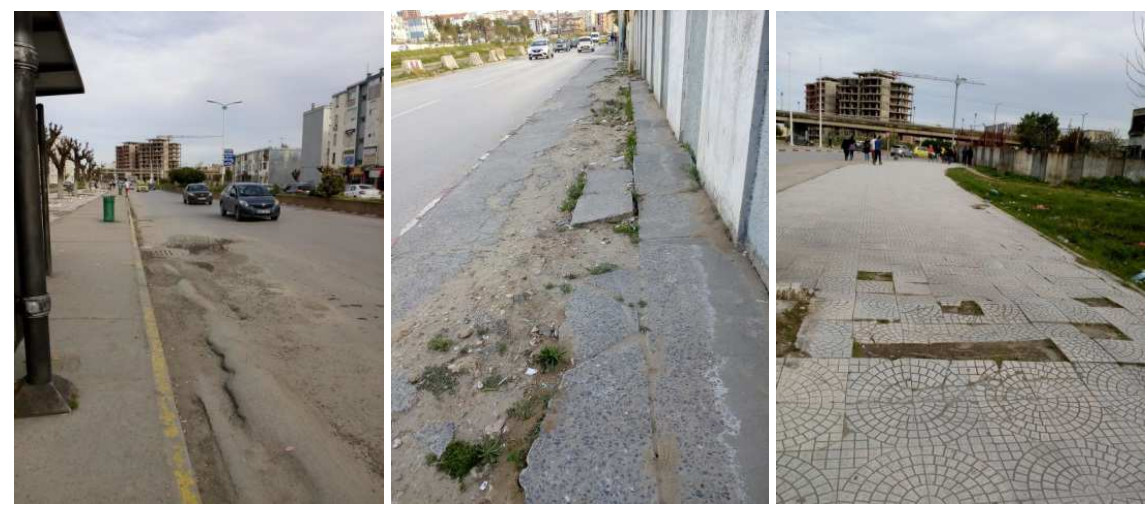

Fig. 5, 6 et 7. Des malfaçons dans la réalisation de la chaussée et ses accessoires

(Auteur, 2018 et 2019)

Si l'on se réfère aux règles techniques, on peut admettre que la chaussée est soumise à différentes contraintes entrainant des détériorations avérées. Suivant de cette logique, le guide de mise en œuvre élaboré par CNERIB précise que la couche de roulement (en tant que revêtement superficiel de la chaussée) est soumise à certains efforts une fois en contact avec la roue. La répétition de ces derniers risques de provoquer des dommages qui compromettent les objectifs de sécurité et du confort. Pour l'efficacité de ce revêtement, le CNERIB recommande sa réalisation en dehors des périodes d'intempéries par l'utilisation des matériaux lui permettant de se doter de 
bonnes qualités de surface. En revanche, les matériaux doivent être choisis de manière à assurer une voirie qui réponde aux qualités normatives.

D'autre part, les trottoirs comme accessoires sont destinés aux piétons en permettant de les sécuriser. Conformément aux recommandations normatives requises, leur réalisation, doit être adaptée aux caractéristiques de la voirie, à la localisation et à l'importance du flux piétonnier. L'enquête entreprise avec les acteurs publics de la DUAC a dévoilé que les travaux de revêtement sont le plus souvent entrepris avant le passage des réseaux enterrés. Et que les matériaux retenus pour les revêtements des trottoirs et voies piétonnes ne sont pas choisis pour leurs caractéristiques mécaniques et esthétiques ni même pour assurer une bonne intégration dans l'environnement paysager. Pour que l'espace soit praticable, les trottoirs, les zones piétonnes ou les traversées de chaussée doivent s'adapter à tous les usagers. Toutefois, et hormis l'aire de détente de la cité 400 logements, l'aménagement de certains espaces observés n'a pas été opéré de manière à insérer des personnes aux besoins spécifiques. Pour ce faire, des déclivités sont à respecter pour rendre l'espace public accessible aux personnes utilisant le fauteuil roulant, la poussette et le chariot, etc. Le guide normatif établi par le CNERIB préconise une déclivité transversale n'excédant pas $2 \%$, et une déclivité axiale maximale des rampes inférieure à $4 \%$ sur des tronçons de $20 \mathrm{~m}$ séparés par des paliers horizontaux d'au moins 1,40 m de largeur.

\subsection{Rénovation du réseau d'assainissement et d'eau potable}

Annaba est une ville située à un niveau inférieur à celui de la mer, une situation favorisant le risque d'inondations de certains quartiers de la ville dont la plaine Ouest et Oued d'heb. Ce phénomène dont les effets sont préjudiciables à l'environnement et la santé publique, est liéà plusieurs facteurs. En effet, les aléas climatiques, les particularités topographiques, géologiques et hydrodynamiques ainsi que la géomorphologie des bassins font des parties Nord et Ouest de la ville des zones susceptibles à la stagnation des eaux et à l'accumulation des sédiments en période de crue.Il est important de rappeler que depuis les inondations du 11 novembre 1982 ayant causé des dégâts matériels et humains importants, un vaste programme d'aménagement hydraulique a été mis en œuvre pour réduire la menace d'inondation qui pesait sur la ville. Toutefois, selon la direction de l'hydraulique (2002), les intempéries de 2002 ont montré d'autres carencesliées essentiellement à l'enfoncement des principaux émissaires par les charriages de matériaux provenant de l'érosion du massif de $1^{\prime} E d o u g h{ }^{8}$ et des chantiers de construction dans les contreforts de ces massifs; à la réduction de la section des oueds Bouhdid et Sidi-Harb ${ }^{9}$ envasés se traduisant

8 C'est un mont d'environ $1000 \mathrm{~m}$ d'altitude, caractérisé par un relief fortement accidenté et relativement arrosé (1200 à $1300 \mathrm{~mm} / \mathrm{an})$.

9 Ces deux oueds traversent la partie ouest de la ville où est située la cité plaine ouest. 
par de fréquents débordements; et enfin à l'obturation complète de la galerie de drainage passant sous l'hôpital Ibn Rochd qui constitueune menace pour le centre hospitalier et la partie basse de la ville. Il est possible de croire que l'obstruction des cours d'eaux a constitué une contrainte à l'évacuation des eaux de crues, et a engendré des débordements d'oueds et l'inondation des quartiers situés notamment dans la partie Sud et Ouest de la ville.

D'autres facteurs tels que l'accroissement de la population et le développement accéléré du nombre de logements à Annaba ont rendu obsolète le réseau d'assainissement mis en place depuis la colonisation. La rénovation de ce réseau (quasiment obstrué) s'est avérée incontournable pour les autorités locales dont l'intervention s'accomplissait le plus souvent dans des conditions pénibles et avec un matériel inadéquat. Une enveloppe de 160 milliards de centimes a été consacrée à cette opération axée sur le redimensionnement des conduites (dépassant $1 \mathrm{~m}$ de diamètre) pour évacuer les eaux, éviterl'accumulation et la formation des flaques et réduire considérablement les fuites (Rahmani, 2009).

A l'échelle du quartier, une première étude du réseau d'assainissementde la cité Plaine-Ouest élaborée par l'Entreprise Nationale des Projets Hydraulique (1996), a décelé un problème de fonctionnement et de dimensionnement des collecteurs étroitement lié aux réseaux eaux usées et eaux pluviales. Cette étude a montré que le réseau eaux usée connaissait un sous-dimensionnement au vu des extensions qui y sont raccordées. Sachant qu'il n'existe pas de collecteur principal central ou latéral permettant la collecte directe des eaux usées et leur évacuation vers les stations de relevage appropriées. Les dépôts solides s'amassant dans les regards et le manque d'entretien du réseau, influent sur l'écoulement rapide des effluents. Relativement auréseaueaux pluviales et ménagères, celui-ci concerne les eaux pluviales provenant de toitures, voiries, parkings, et du ruissellement, recueillies par des collecteurs au moyen des avaloirs et bouches à grilles. Le rejet se fait dans les Oueds les plus proches (Bouhdid, Oued-Eddheb, Oued-Forcha, Canal de ceinture). A vrai dire ce système d'évacuation se trouve insuffisant au moment des grandes crues en raison du manque d'entretien du réseau et de son sous-dimensionnement. L'on remarque ainsi une forte stagnation d'eau dans les parkings, chaussées, et parfois même à l'entrée des immeubles.

C'est dans cette perspective que s'inscrit l'opération d'amélioration urbaine. A l'issue des travaux entrepris, les personnes interrogées ${ }^{10} \mathrm{~s}^{\text {'accordent toutes sur }}$ l'acquisition d'une nouvelle image allant d'un quartier insalubre à un quartier salubre. Pour elles, l'opération d'amélioration urbaine a véritablement changé l'image des quartiers populaires qui deviennent plus propres et plus attractifs. Cette opinion est illustrée à travers l'exemple de la cité 1276 logements

10 Il s'agit des acteurs publics, enseignants, fonctionnaires, cadres de l'Etat, commerçants, chômeurs, etc. 
submergée (en 2004) par les eaux de pluies (Figure 8). La cité retrouve petit à petit sa salubrité après la rénovation du réseau d'assainissement (Figure 9 et 10).
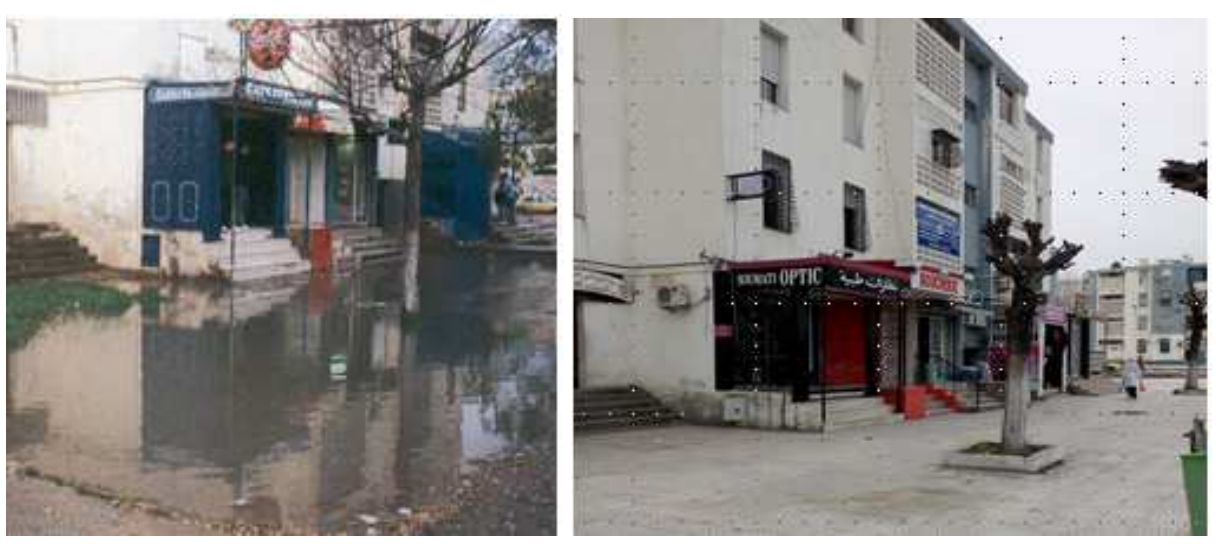

Fig. 8. Cité 1276 logements Fig. 9. Cité 1276 logements «Boulevard Djemouai Mohammed » «Boulevard Djemouai Mohammed » Avant (2004) Actuellement (2018)

Source : Mebirouk et Chikh, 2004, Auteur, 2018

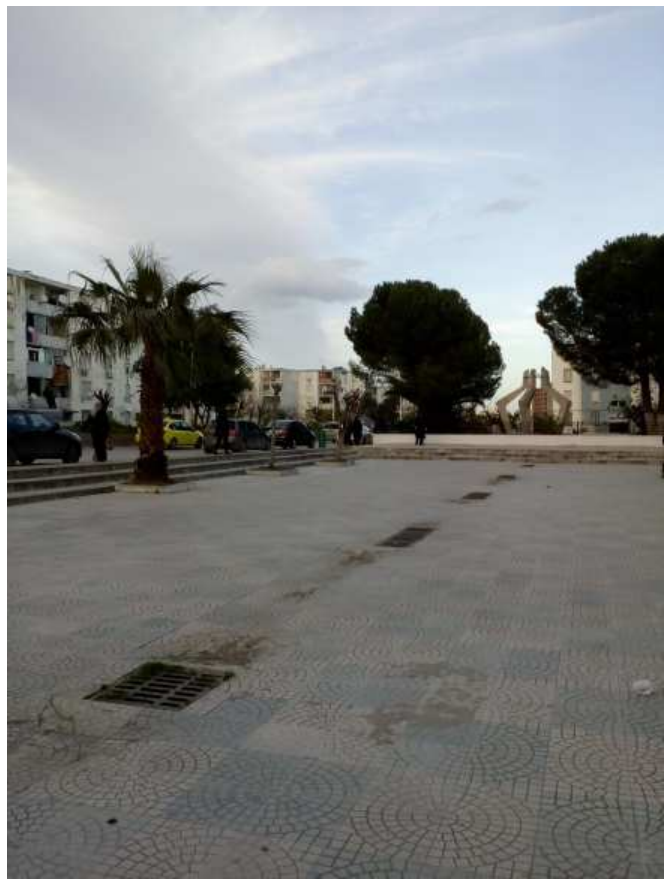

Fig. 10. Redimensionnement du réseau d'assainissement Source : Auteur, 2018 
L'AMÉLIORATION URBAINE, UNE ACTION PLURIELLE POUR RECHERCHER LA QUALITÉ DE VIE. QUELS EFFETS SUR LA POPULATION ET LE CADRE DE VIE DANS LES ENSEMBLES COLLECTIFS.

\subsection{Création des espaces verts et réalisation du mobilier urbain}

La présence de la nature en milieu urbain représente une composante assez importante pour la qualité du cadre de vie grâce à la diversité de ses fonctions: sociale, psychologique, paysagère et politique: production de l'agriculture et de la forêt, préservation des ressources humaines et naturelles (flore et faune), ouverture au public pour le divertissement, la détente, l’oxygénation, les loisirs (Lamri, 2012, Mebirouk, 2008). Par ailleurs, si l'Organisation Mondiale de la Santé (OMS) a recommandé pour chaque citadin environ $10 \mathrm{~m}^{2}$ de couverture végétale, c'est pour lui procurer du bien-être et de l'épanouissement. Cette exigence normative n'est pas encore respectée pour le cas algérien, les normes ministérielles (décrétées depuis les années 1970, et toujours en vigueur) préconisent pour un habitant entre ( 1 et $1,5 \mathrm{~m}^{2} /$ habitant) de verdure, ce qui témoigne de l'insuffisance de ces espaces dans les cités collectives algériennes. Le regain d'intérêt au paysage végétal est apparu avec la promulgation de la loi ${ }^{\circ} 07-06$ du 3 Mai 2007 relatif à la gestion, à la protection et au développement des espaces verts. Et c'est ainsi, que l'introduction des espaces verts dans tout projet de construction devient une obligation que doit prendre en charge toute étude urbanistique et architecturale publiques et privées. Cette loi a énoncé les biens faits de l'espace vert comme élément essentiel pouvant déterminer la qualité de l'environnement urbain. L'espace vert peut répondre aux besoins physiques et psychologiques de l'homme, protéger et rehausser les ressources naturelles de base (air, eau, sol, végétation, animaux); et contribuer au développement économique (Lamri, 2012: 7). Le choix des végétaux est en revanche conditionné par la nature du sol et celle du climat. La fonction de ces végétaux est l'épuration des quartiers de la ville, l'absorption des eaux pluviales, la fixation des pollutions atmosphériques, la régulation de température et la création des zones d'ombres pour les usagers. Un bon aménagement d'espaces urbains publics passe, selon les recommandations du CNERIB (2007) par la compréhension du paysage et ses données techniques à savoir l'ensoleillement, la topographie du site, l'ambiance acoustique, la capacité de desserte, la nature du sol et les conditions climatiques.

La création et l'aménagement des espaces verts dans certains quartiers, dans le cadre de cette politique, a été accompagnée d'équipements d'agréments nécessaires pour les usagers tels que: bancs de repos; aires de jeux dont le revêtement de sol nécessite une dalles ou pavés en béton habillés d'un revêtement souple afin de protéger l'enfant des éventuelles blessures; et éclairages publics permettant la perception des obstacles, l'identification des personnes à une dizaine de mètres et l'épanouissement nocturne des cités (Figures 11 et 12). 


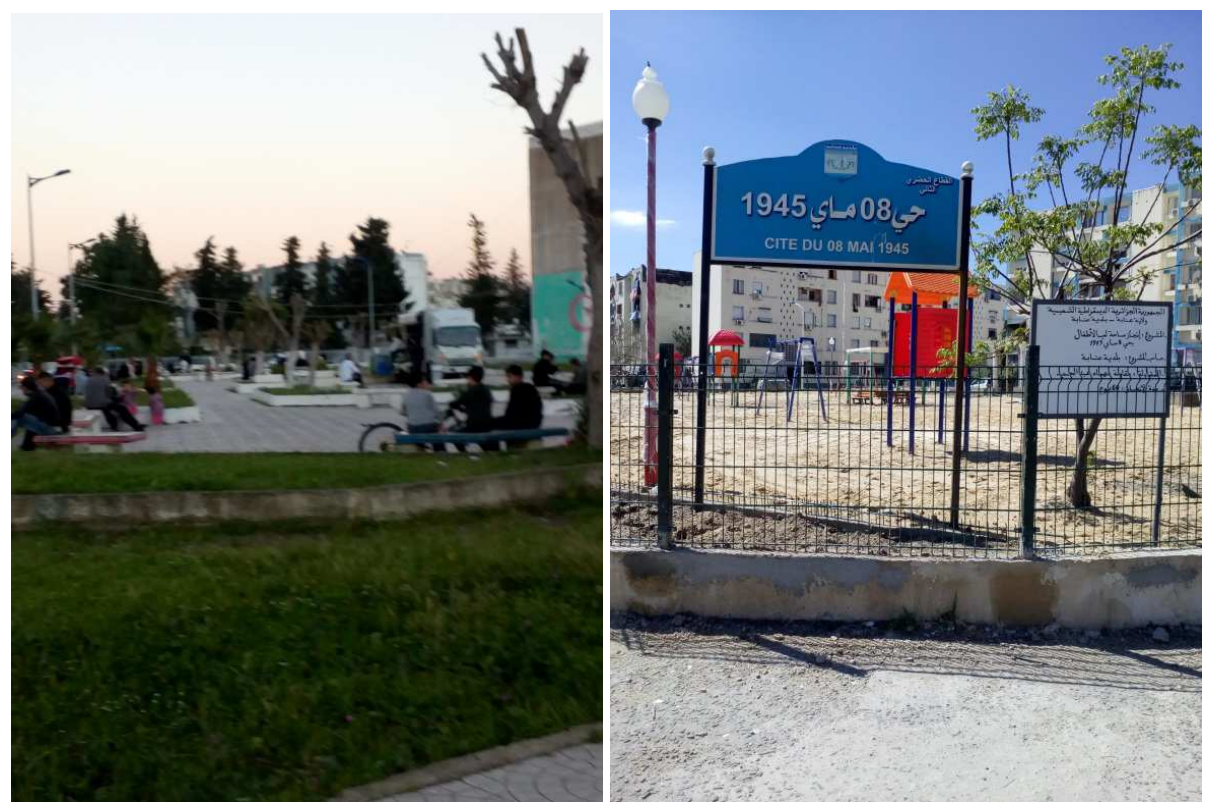

Fig. 11. Introduction de l'espace vert Fig. 12. Création des aires de jeux clôturées dans la cité 400 logements (cité 08 mai 1945)

Source : Auteur, 2018

L'ensemble des objets et dispositifs publics installés dans l'espace extérieur tend à remplir diverses fonctions : sécurité des piétons, confort, embellissement, propreté ou signalétique.

Les axes structurants des cités concernées par ces opérations urbaines sont dotés debancs qui n'ont pas été programmés en se rapportant la taille de la population de la ZHUN abritant d'après le recensement de 2008, environ la moitié de population totale (304 749hab). En plus des abris pour bus, les quartiers en question sont pourvus de corbeilles pour papiers, mais ces dernières restent sous-utilisées par une population encore peu sensibilisée au respect de l'environnement. Il convient ici de mentionner que les cabines téléphoniques ont été également réalisées mais ultérieurement supprimées à cause de l'usage du téléphone portable. Ce qu'il faut relever comme insuffisance, c'est le plan de repérage, le balisage et la programmation des espaces de détente destinés à la population du troisième âge. 


\section{Confort etcréation d'un environnement agréable}

\subsection{Un pas vers l'intégration sociale}

Parmi les quartiers marginaux d'Annaba, nous pouvons mentionner l'exemple de la Cité 600 logements (située à la Plaine Ouest) dont les habitants sont des sinistrés provenant du bidonville d'Oued-D'heb suite aux inondations de 1982. Le processus de relogement a poursuivi les habitants comme attribut stigmatisant car il s'agit d'une population «bidonvilloise» vivant dans des conditions de vie pénibles et provenant (à l'origine) des villes limitrophes dans le cadre de l'exode rural. Selon l'enquête menée avec les habitants relogés, ce quartier, préalablement destiné aux militaires de la région de Annaba, a été déconsidéré en portant un toponyme dévalorisant «Bengladesh» pour justifier et légitimer les comportements et attitudes d'une population dite du deuxième rang : non-citadine et rurale.

L'isolement et le rejet vécus par les habitants pourraient faire naitre de l'hostilité pour un quartier dégradé où l'on ne peut échapper à l'insécurité, pourtant la population reste très attachée à sa cité. Les jeunes interrogés nous disent «nous aimons notre quartier, et nous y restons », mais ils déclinent ce toponyme «quartier Bengladesh » qui est, selon eux, synonyme d'incivisme et de décadence.

Les travaux d'exécution de l'opération urbaine prévus en 2004 par l'Office de Promotion et de Gestion Immobilière «OPGI (peinture des immeubles, étanchéité et infiltration d'eau) ainsi que les opérations d'aménagement des espaces extérieurs n'ont pas vu le jour. C'est seulement au mois de mars 2018 que la réhabilitation urbaine s'amorce par l'intervention sur deux équipements de proximité : l'un socioculturel (Mosquée) et l'autre sportif (terrain de proximité) (Figure 13 et 14); et par la création ou la rénovation des voies piétonnes (Figure 15). La requalification de ces équipements est perçue comme un pas vers le désenclavement du quartier qui développe une certaine attractivité en captivant une population venant de tous les quartiers limitrophes pour pratiquer du sport ou faire de la prière.

Au vu de cette amélioration, le président du comité de quartier conteste le rythme relativement faible de l'exécution des travaux en remettant en cause les compétences de l'entreprise désignée à cet effet.

Obstinée par l'insertion totale de son quartier et l'amélioration de son image, la population résidente a cotisé une somme d'argent conséquente ${ }^{11}$ pour réaliser des travaux de salubrité indispensables pour la qualité de vie des habitants. Initiée sans l'appui des pouvoirs publics, cette action respectueuse de l'environnement a porté sur la collecte des déchets et la désobstruction des

\footnotetext{
11 La somme d'argent n'a pas été définie par le président du comité du quartier.
} 
égouts occasionnant des inondations menaçantes ${ }^{12}$. Par ces actions volontaristes, les jeunes essayent d'affirmer le mérite d'un nouveau toponyme « quartier Suisse » à la place de « Bangladesh » que les pouvoirs locaux leur ont promis il y a environ 15 ans $(2004)^{13}$.
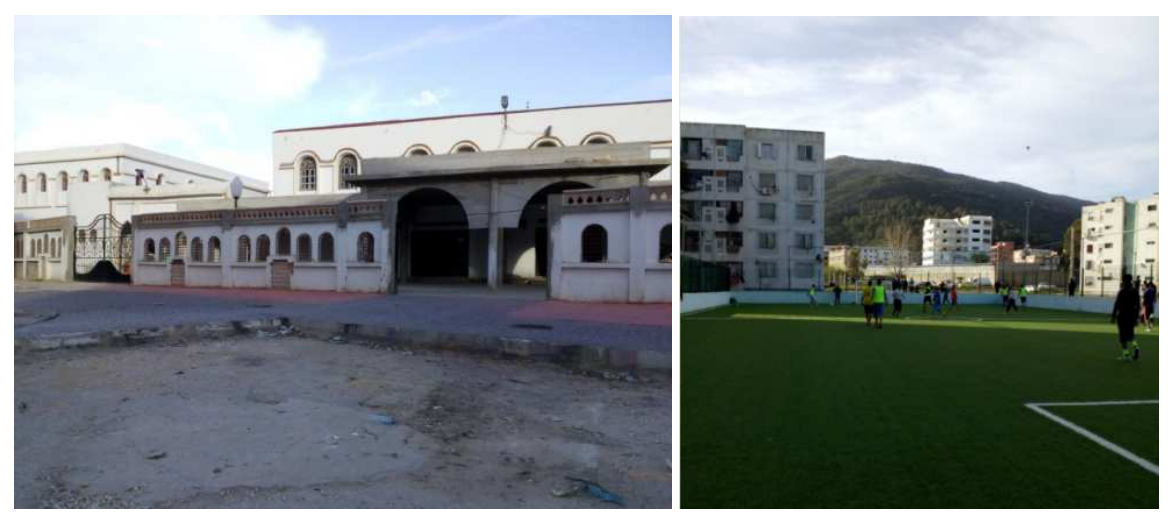

Fig. 13 et 14. Vers une intégration urbaine par la réhabilitation de la mosquée et le terrain de proximité (Auteur, 2018)

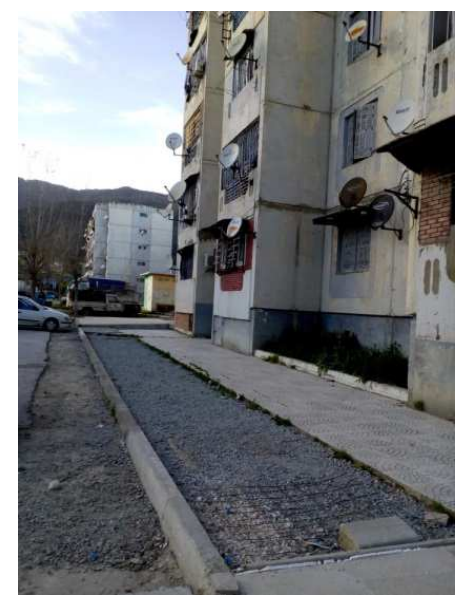

Fig. 15. De la rénovation à la création des trottoirs et voies piétonnes (Auteur, 2018)

2.2. Des alternatives au besoin spatial par certaines catégories de population

La programmation des espaces de détente destinés aux différentes catégories d'âge a toujours été problématique notamment dans les ensembles de

12 L'argent cotisé a permis d'acheter des dépotoirs pour la collecte et le tri des déchets ainsi que les outils et ustensiles nécessaires pour déboucher les égouts.

13 C'est la déclaration du responsable de l'OPGI « secteur Plaine-Ouest ». 
logements collectifs où le confort élémentaire était pratiquement inexistant. Avec ce projet de requalification urbaine qui est avant tout social, la population du troisième âge (sexe confondu) ne trouve pas encore d'espace spécifique lui permettant d'exercer une pratique socio-spatiale remontant aux années 1980. En effet, depuis la réalisation des ensembles collectifs à Annaba, les personnes âgées font de l'espace extérieur (espaces attenants aux logements voire même des terrains vagues non définis) le lieu propice de regroupement et de rencontre quotidiens (Figure 16).

Peu importe la saison ou ses conditions climatiques, les femmes âgées de la Plaine ouest particulièrement des cités (400 logements, 600 logements et 1276 logements) tiennent coute que coute à ces rencontres pour échanger des informations et notamment évoquer des souvenirs. Elles sont même avivées par l'appropriation d'escaliers situés au pied de locaux commerciaux (Figure 17), l'essentiel pour ces femmes interrogées est que «l'espace convoité soit moins visible et notamment moins fréquenté par la population résidente ».

Le groupement massif dans des lieux et espaces inappropriés est devenu une pratique usuelle. L'appropriation des lieux publics par cette catégorie peut être déchiffrée comme revendication d'un «besoin spatial » qu'elle adresse aux acteurs publics en général et aux professionnels en particulier et ce afin de connaitre les aspirations de chacun en utilisant incontestablement l'outil de concertation et par voie de conséquence intégrer dans les conceptions d'aménagement des espaces destinés à chaque catégorie d'âge.

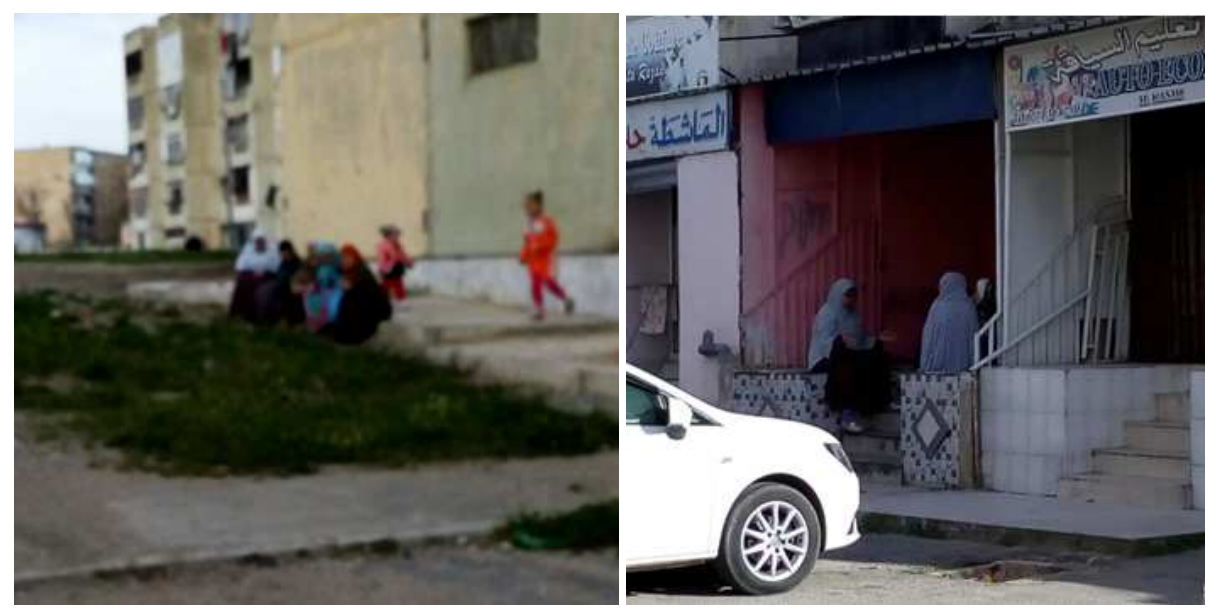

Fig. 16 et 17. De 1985 à 2018, la rencontre des femmes âgées est omniprésente dans différents quartiers Cité 600 logements Cité 1276 logements Source: Auteur, 2018 


\section{Discusion}

L'analyse du produit fini montreque le plan d'amélioration urbaine a contribué à l'évolution du cadre de vie de plusieurs quartiers. Mais avec un projet aussi ambitieux et global, l'intervention se limite aux axes structurants. En effet, ce n'est pas dû au manque de budget mais essentiellementà sa gestion que certains fragmentsdes cités 1172 logements, 600 logements, 400 logements, oued Edheb, se trouvent soit totalement exclus de l'opération (Figures 18 et 19), soit partiellement exclus : une intervention rudimentaireréduite au revêtement de la chaussée (Figures 20 et 21).

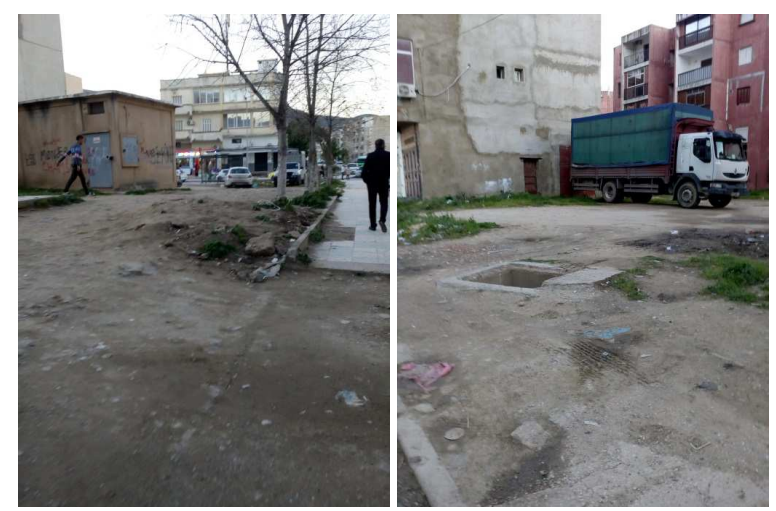

Fig. 18 et 19. Exemple d'un espace extérieur exclu de l'opération d'amélioration urbaine. La cité 400 logements « Plaine-Ouest » (Auteur, 2018)

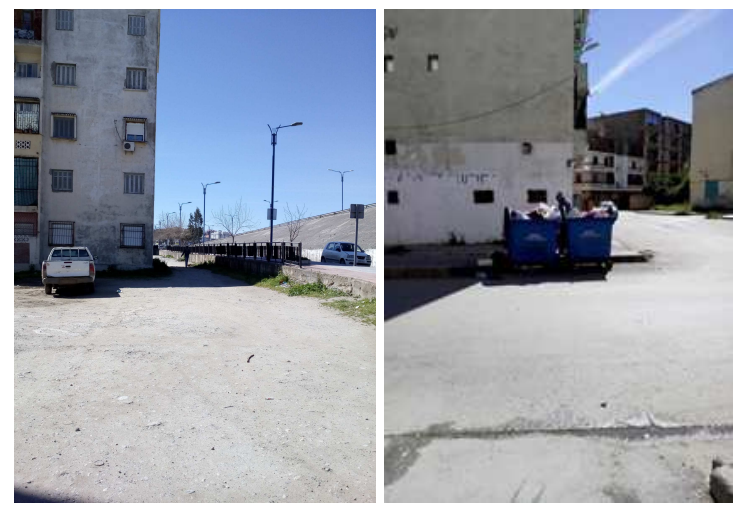

Fig. 20 et 21. Aménagement rudimentaire d'un espace public de la Cité Oued-Edheb limité au revêtemnt de la chaussée (Auteur, 2018) 
Aussi, la qualité d'exécution de ces opérations s'est révélée peu satisfaisante compte tenu de certaines imperfections constatées par les administrations locales dans certains sites (Benachour K, 2011). Nous citons à titre d'exemple : des chaussées mal-réalisées, des trottoirs hors normes et inadaptés aux différentes utilisations, une végétation choisie aléatoirement, et un mobilier urbain insuffisant ou incomplet. Par ailleurs, les carences relevées sont liées aux entreprises spécialisées et aux partenaires respectant les cahiers des charges de l'opération d'amélioration urbaine. Le programme en question exige qu'une étude fondée sur la réalité du terrain soit menée afin de respecter la conformité avec le plan de restructuration et de modernisation des centres urbains. Le résultat d'une opération urbaine est étroitement lié au choix de bureaux d'études, à un suivi de chantier régulier et principalement à la participation du citoyen. L'amélioration urbaine est une action plurielle dont les acteurs sont les collectivités locales, les directions déconcentrées, les opérateurs économiques ainsi que la société civile. Les habitants des cités requalifiées s'attendaient à une implication réelle et non à une simple information sans que leurs avis ne soient pris en compte dans le processus de conception. L'implication du citoyen se limite-t-elle à une simple procédure d'information si l'on sache que l'amélioration urbaine vise à réintégrer les habitants dans le processus de production de leur cadre de vie ?

La réponse est forcément négative, l'implication est un processus de construction collective qui se poursuit jusqu'à la réalisation du projet et même au-delà si nécessaire. Ceci renvoie à la notion de concertation qui constitue une démarche par laquelle est organisée la participation des citoyens, directement ou à travers des instances représentatives, au processus de décision. La finalité de cet outil est d'améliorer réellement la participation du public à la conception des projets, y compris lorsque celle-ci est déjà exigée par des dispositions tant législatives que réglementaires. Il faut rappeler que la démarche concertée comme cadre général d'orientation rejoint les nouveaux objectifs de l'État Algérien afin d'inciter le citoyen à réfléchir son quotidien social et urbain et à agir davantage en ce sens.

\section{Conclusions}

Les opérations d'amélioration urbaine ont réellement traduit la volonté de l'État de réhabiliter l'image des quartiers dégradés et d'y rechercher la qualité de vie synonyme du bien-être social. Seulement, pour que ces actions puissent aboutir, il faut nécessairement mobiliser les compétences en alliant l'ensemble des forces intellectuelles intervenant dans le processus de l'amélioration urbaine, et aussi se rapprocher de la population qui doit se motiver par le projet pour réussir son cadre de vie. 
L'opération d'amélioration urbaine n'est pas uniquement une exigence technique mais surtout une compétence et une main d'œuvre qualifiée. Pour une amélioration réelle du tissu urbain, les autorités publiques, les élus, les professionnelset également les citoyens sont appelés à assumer pleinement leurs responsabilités. L'État algérien est conscient qu'aucun résultat probant en matière de développement, s'il se veut durable, ne peut se concrétiser qu'à travers la participation de toutes les parties prenantes. En l'absence de négociation, les études ne peuvent révéler les attentes et aspiration des habitants et citoyens. La concertation et le débat public sont aujourd'hui les instruments de réflexion collective, et de construction des politiques et actions durables. La démarche concertée est plus qu'un paradigme, c'est un instrument à mettre en œuvre dans toute opération urbaine afin de garantir sa réussite.

\section{BIBLIOGRAPHIE}

Bahri, R., 2014, Intervention sur les espaces extérieurs dans les grands ensembles, Cas d'El-Bouni, Mémoire de Master, Département d'Architecture. Université Badji Mokhtar Annaba.

Barbarino, N., 2005, De la qualité de vie au diagnostic urbain, vers une nouvelle méthode d'évaluation. Le cas de la ville de Lyon, Thèse de Doctorat en Géographie. Université Lumière Lyon 02.

Benachour, K., 2011, Constantine : Plan d'amélioration urbaine Fini l'amateurisme, Horizon quotidien national d'information du 23 Mai.

El-Annabi, 2007, Deux opérations d'amélioration urbaine à Annaba, le quotidien du 14 Aout.

El-Kadi, G., 1997, Qualité de vie et habitat précaire dans quelques pays du monde arabe, Revue Espace, Populations, Sociétés, 1997-1. Les populations du monde arabe, pp. 35-47.

Lamri, S., 2012, Espace vert urbain et périurbain de Sétif : état des lieux et place dans la gestion municipale, Mémoire de Magister, Département de Biologie Ecologie Végétale, Université FARHAT ABBAS - Sétif.

Mebirouk, H., 2002, Les espaces publics dans les grands ensembles : rapport entre espaces conçus et espaces vécus. Cas de Annaba, Mémoire de Magister, Université de Mentouri Constantine, $256 \mathrm{p}$.

Mebirouk, H., 2008, Entretenir, valoriser, et préserver les espaces verts dans les ensembles de logements collectifs à Annaba, in Revue Sciences et Technologie « D » $\mathrm{n}^{\circ} 28$, Université Mentouri Constantine, pp. 41-48.

Mebirouk, H., 2018, Urbanimprovement operations. Evaluation of the new urban habitat area of Annaba, International Journal of Human Settlements, Vol. 2. Nr.1. 2018, pp. 49-59.

Mebirouk, H, Chikh, N., 2004, Les nuisances en milieu urbain, acteurs et effets ; Cas de Annaba, in Séminaire international écologie environnementale urbaine: état actuel et prospectives, Département Science de la nature et de la vie, Université de Constantine, 15 au 17 mai.

Mebirouk, H., Zeghiche, A., Boukhemis, K., 2005, Appropriation de l'espace public dans les ensembles de logements collectifs, forme d'adaptabilité ou contournement de normes ? Cas des ZHUN d'Annaba, in revue Norois, $\mathrm{n}^{\circ}$ 195, Presses Universitaires de Rennes, pp. 59-77.

Navez-Bouchanine, F., 1992, Les espaces publics dans les villes marocaines, Les Annales de la Recherche Urbaine, $\mathrm{n}^{\circ}$ 57-58.

www.urbanisme.equipement.gouv.fr/cdu/datas/annales/boucha.htm. 
Rahmani, M., 2009, Rénovation du réseau d'assainissement à Annaba Les canalisations sont vieilles et souvent obstruées, La Tribune du 05 Janvier 2009.

Sablet, M. De, 1992, Des espaces urbains agréables à vivre Place, Rues, Squares et jardins, Paris, édition Le Moniteur. Paris.

Sidi-Salah-Nasri, Z., 2010, La gouvernance urbaine Une démarche incontournable pour un habiter durable. Cas de l'amélioration urbaine de la cité Zouaghi à Constantine, Mémoire de Magister, Université de Constantine.

Zahi, N., 2012, Les retombées de l'amélioration urbaine sur le cadre de vie par la réhabilitation des quartiers de la plaine-ouest de la ville d'Annaba (Nord-Est Algérien), Mémoire de Magister, Département d'Aménagement, Université BADJI Mokhtar Annaba.

*** Centre National d'Etudes et Recherches Intégrées du Bâtiment (CNERIB) (2007), Guide de mise en œuvre des travaux d'amélioration urbaine, Ministère de l'Habitat et de l'Urbanisme. Alger.

*** Décret exécutif $\mathrm{n}^{\circ} 13-13$ du 15 janvier (2013), fixant les règles d'organisation et de fonctionnement des services extérieurs du ministère de l'habitat et de l'urbanisme.

*** Direction de l'Urbanisme et de la Construction d'Annaba (DUC), (2010 (a)), État des sites pris en charge en étude. Situation arrêtée le 11 Février.

*** Direction de l'Urbanisme et de la Construction d'Annaba (DUC), (2010 (b)), État des sites pris en charge en travaux. Situation arrêtée au 11 Février.

*** Direction de l'Hydraulique, Rapport sur la protection contre les inondations des agglomérations vulnérables de la wilaya, Annaba

*** Entreprise Nationale des Projets Hydrauliques De L'est (ENPH), 1996, Etude de l'assainissement de la Plaine-Ouest, phase II, Mesures d'améliorations immédiates, Annaba.

*** Instruction ministérielle $\mathrm{n}^{\circ} 89$ du 24 juin 1995 relative à la décentralisation de la gestion des opérations d'études et d'aménagement relevant des chapitres 711 et 721 .

*** Loi $\mathrm{n}^{\circ}$ 07-06 du 13 Mai 2007 relative à la gestion, à la protection et au développementdes espaces verts.

*** Loi ${ }^{\circ}$ 06-06 du 20 Février 2006 portant loi d'orientation de la ville.

*** Loi n 90-09 du 07 Avril 1990 relative à la Wilaya. 